\title{
Genistein inhibits invasion and migration of colon cancer cells by recovering WIF1 expression
}

\author{
JIE ZHU, JUN REN and LIMING TANG \\ Department of Gastrointestinal Surgery, Changzhou No. 2 People's Hospital, \\ Nanjing Medical University, Changzhou, Jiangsu 213000, P.R. China
}

Received May 5, 2017; Accepted November 7, 2017

DOI: $10.3892 / \mathrm{mmr} .2018 .8760$

\begin{abstract}
Colon cancer is characterized by invasion and migration. DNA methylation of $\mathrm{CpG}$ islands in tumor suppressor genes is considered to be an epigenetic mechanism underlying cancer development. Epigenetic silencing of a gene may be reversed by drugs, including genistein. The present study aimed to determine the effect of genistein on Wnt inhibitory factor 1 (WIF1) and invasion, and migration of colon cancer cells. The viability of HT29 colon cancer cells was suppressed by genistein in a dose dependent manner. Following $72 \mathrm{~h}$ of treatment with 10,20 and $60 \mu \mathrm{mol} / 1$ genistein, increased demethylation of WIF1 was induced in a dose-dependent manner. Additionally, the invasive/migratory abilities of cells treated with genistein decreased in a dose-dependent manner. Reverse transcription-quantitative polymerase chain reaction and western blot analyses were performed to identify the mRNA and protein expression levels of invasion/migration-associated factors. Following treatment with genistein, matrix metalloproteinase (MMP) 2 and MMP9 expression levels decreased, whereas the expression of metalloproteinase inhibitor 1 and E-cadherin increased significantly. In addition, the expression levels of proto-oncogene Wnt- 1 (Wnt-1)/ $\beta$-catenin pathway-associated factors, $\beta$-catenin, c-Myc proto-oncogene protein and cyclin D1 decreased in a dose-dependent manner following treatment with genistein. The invasive/migratory abilities of cells transfected with WIF1-small interfering (si) RNA, and those transfected with WIF1-siRNA and treated with genistein, increased notably compared with the control group. The present study demonstrated that genistein was able to inhibit the cell invasion and migration of colon cancer cells by inducing demethylation, and recovering the activity
\end{abstract}

Correspondence to: Dr Liming Tang, Department of Gastrointestinal Surgery, Changzhou No. 2 People's Hospital, Nanjing Medical University, 68 Gehu Middle Road, Changzhou, Jiangsu 213000, P.R. China

E-mail: erx8524@126.com

Key words: genistein, Wnt inhibitory factor 1, methylation, invasion, migration, colon cancer of WIF1 by altering the expression of invasion-associated factors, and components of the Wnt signaling pathway.

\section{Introduction}

Colon cancer is one of the most common malignant carcinomas, the morbidity of which is the second highest among gastroenteric tumors worldwide (1). The majority of patients with colon cancer suffer from hemafecia, diarrhea and pain. Colon cancer affects human health. Due to the late stage of diagnosis and poor efficacy of treatment, the incidence and mortality rates are $8-9 \%$ (2). The 5-year survival rate of early-stage colon cancer exceeds $70-90 \%$, although the survival rate of advanced colon cancer is unsatisfactory. National Comprehensive Cancer Network guidelines recommend surgical treatment for patients with primary-stage colon cancer. However, in patients with advanced-stage disease, it is challenging to completely remove the tumor via surgical procedures due to the ubiquitous invasion and migration. Therefore, palliative surgery combined with chemotherapy is more frequently applied (3). Chemotherapy is given priority among comprehensive treatments (4). However, anti-drug sensitivity or severe adverse reactions occasionally limit the clinical effectiveness. It is therefore necessary to develop novel drugs exhibiting fewer anti-drug sensitivity effects and adverse reactions. Genistein is a potential drug candidate for the treatment of colon cancer.

The initiation and progression of carcinogenesis occurs when genetic and epigenetic modifications accumulate and interact. DNA methylation of $\mathrm{CpG}$ islands in the promoter region of tumor suppressor genes is considered to be an epigenetic mechanism underlying cancer development $(4,5)$. As a highly conserved metabolism-associated pathway, the Wnt signaling pathway is involved in metabolic processes, including embryonic development, cell proliferation, metastasis and differentiation. The Wnt signaling pathway is inactive in normal cells, and the abnormal activation may induce tumor development. Wnt inhibitory factor 1 (WIF1) is a tumor suppressor, which interacts with Wnt protein to inhibit the canonical and non-canonical Wnt pathways. It has been reported that silencing of WIF1 via methylation is associated with cancers, such as colon cancer (6). A previous study additionally reported that WIF1 inhibited the Wnt signaling pathway by inhibiting $\beta$-catenin accumulation, suppressed esophageal cancer and decreased the expression of E2F 
transcription factor 1, cyclin D1 and c-Myc proto-oncogene protein (c-Myc) to promote the apoptosis of hepatoma cells (7).

Recent studies demonstrated that methylation-mediated epigenetic silencing of genes may be reversed by drugs $(8,9)$. Genistein, a soy-derived isoflavone, is reported to upregulate the mRNA expression of a number of tumor suppressor genes, to antagonize function of growth stimulating factors and to inhibit cellular malignancy. Genistein has a broad-spectrum anti-cancer effect on breast, prostate, esophageal, pancreatic, gastric and colon cancer, and metrocarcinoma, lymphoma and neuroblastoma; and therefore, it is becoming a novel therapy for the treatment of tumors (10-12). However, whether genistein is able to upregulate the mRNA expression of WIF1 through demethylation in colon cancer remains to be elucidated.

The present study investigated the effect of treatment with genistein on WIF1 in colon cancer cells, particularly on cell invasion and migration. The present study suggested a mechanism underlying the genistein-mediated demethylation of the WIF1 promoter region, and its influence on downstream molecular factors in the associated pathways. The results of the present study may contribute to the treatment of colon cancer.

\section{Materials and methods}

Patients and tissue samples. Written informed consent was obtained from all participants prior to the present study. A total of 52 patients (36-79 years old; median age, 55 years), 32 males and 20 females, with colon cancer from Changzhou No. 2 People's Hospital (Changzhou, China) were included in the present study, from November 2015 to November 2016. The present study included two patients with well-differentiated tumors, 31 patients with moderately-differentiated tumors and 19 patients with poorly-differentiated tumors. Of those patients, a total of nine were in the $\mathrm{T} 1+\mathrm{T} 2$ stage, 22 patients were in the T3 stage and 21 patients were in the T4 stage. Preoperative clinical and pathological follow-up data were completed by all patients. All tissue samples of patients were collected according to procedures approved by the institutional review board of the independent ethics committee of Changzhou No. 2 People's Hospital. Adjacent normal colon tissues were also collected as negative controls. Reverse transcription-quantitative polymerase chain reaction (RT-qPCR) and western blot analyses were performed to detect the mRNA and protein expression levels of WIF1 in colon cancer tissues and adjacent normal colon tissues. Detailed procedures are described in the designated sections below.

Cell culture. Human colon mucosal epithelial cells NCM460 and colon cancer cell lines (HT29, SW620, LOVO, HCT116) were purchased from the American Type Culture Collection (Manassas, VA, USA) and cultured in RPMI 1640 medium (Gibco; Thermo Fisher Scientific, Inc., Waltham, MA, USA) containing $10 \%$ fetal bovine serum (FBS; Gibco; Thermo Fisher Scientific, Inc.) with $1 \%$ penicillin/streptomycin (Invitrogen; Thermo Fisher Scientific, Inc.) at $37^{\circ} \mathrm{C}$ with $5 \% \mathrm{CO}_{2}$. Cells in the logarithmic phase were used for subsequent experimentation.

RT-qPCR and western blot analyses were performed to detect the mRNA and protein expression of WIF1 in the above colon cancer cell lines and the normal colon cell line NCM1460.

Cell viability assay. The effect of genistein on HT29 cell viability was detected using the Cell Counting Kit-8 (CCK8; Beyotime Institute of Biotechnology, Haimen, China). HT29 cells were seeded in 96-well plates at the density of $5 \times 10^{3}$ cells/well and incubated for $24 \mathrm{~h}$ at $37^{\circ} \mathrm{C}$. Genistein (Sigma-Aldrich; Merck KGaA, Darmstadt, Germany) was subsequently added to each well at different final concentrations, including $0,5,10,20,40$ and $60 \mu \mathrm{mol} / 1$ and incubated for $12,24,48$ and $72 \mathrm{~h}$ at $37^{\circ} \mathrm{C}$. Subsequently, $10 \mu \mathrm{l} \mathrm{CCK8}$ solution was added to each well and incubated for a further $4 \mathrm{~h}$ at $37^{\circ} \mathrm{C}$ with $5 \% \mathrm{CO}_{2}$. The optical density (OD) values were measured at a wavelength of $450 \mathrm{~nm}$ using a microplate reader (Thermo Fisher Scientific, Inc.). Data are presented as the ratio of viable cells to control cells.

RT-qPCR and western blot analyses were performed to detect the mRNA and protein expression of WIF1 in HT29 colon cancer cells and genistein-treated cells (10, 20 and $60 \mu \mathrm{mol} / \mathrm{l})$.

Methylation specific PCR (MSP). Following a 72 h-treatment with 10, 20 and $60 \mu \mathrm{mol} / 1$ genistein independently, HT29 cells $\left(1.5 \times 10^{4}\right.$ cells/sample) were subjected to bisulfite conversion using the EZ DNA Methylation-Startup kit (Zymo Research Corp., Irvine, CA, USA), according to the manufacturer's protocol. Subsequently, $30 \mathrm{ng}$ converted DNA of each sample was used for the MSP amplification. The WIF1 promoter region was identified using the online software MethPrimer 2.0 (Chinese Academy of Medical Sciences, Beijing, China), with methylated [(M)] allele-specific primers, forward (F) 5'-CGTTTTATTGGGCGTATCGT-3' and reverse (R) 5'-ACTAACGCGAACGAAATACGA-3'; and unmethylated $[(\mathrm{U})]$ allele-specific primers, F5'-GGGTGT TTTATTGGGTGTATTGT-3' and R 5'-AAAAAAACTAAC ACAAACAAAATACAAAC-3'. MSP amplification was performed using the following thermocycling conditions: Initial denaturation for $1 \mathrm{~min}$ at $94^{\circ} \mathrm{C} ; 35$ (M) or 40 (U) cycles of denaturation at $94^{\circ} \mathrm{C}$ for $1 \mathrm{~min}$, and annealing at $65^{\circ} \mathrm{C}(\mathrm{M})$ or $56^{\circ} \mathrm{C}(\mathrm{U})$ for $30 \mathrm{sec}$; and final extension at $72^{\circ} \mathrm{C}$ for $1 \mathrm{~min}$.

Cell invasion and migration assay. Cell invasion and migration assays were performed using 24-well Transwell chambers with polycarbonate filters (Corning Incorporated, Corning, NY, USA). Matrigel chambers (BD Biosciences, Franklin Lakes, NJ, USA) were applied to evaluate the effect of genistein $(10,20$ and $60 \mu \mathrm{mol} / \mathrm{l})$ on invasiveness, according to the manufacturer's protocol. Cells treated with genistein for $48 \mathrm{~h}$ were collected and resuspended in serum-free medium and transferred to diluted Matrigel chambers $\left(5 \times 10^{4}\right.$ cells/well). The chambers were incubated for $24 \mathrm{~h}$ prior to examination, with the bottom chambers containing culture medium with $10 \%$ FBS. The invaded cells on the lower surface that passed through the filter were stained using $0.1 \%$ crystal violet for $30 \mathrm{~min}$ at room temperature. Finally, invading cells were counted in five randomly-selected high power fields under a light microscope (Olympus Corporation, Tokyo, Japan, magnification, x100) and the cell number was calculated. A 
cell migration assay was performed using the same procedure as described above for the cell invasion assay, although cell medium without Matrigel was used.

RT-qPCR and western blot analyses were performed to detect the mRNA and protein expression of the invasion/migration-associated factors E-cadherin, matrix metalloproteinase (MMP) 2, MMP9, tissue inhibitor of metalloproteinase inhibitor 1 (TIMP1), $\beta$-catenin, $c-M y c$ proto-oncogene protein (c-Myc) and cyclin D1, serving a role in the $\mathrm{Wnt} / \beta$-catenin signaling pathway.

Small interfering (si)RNA transfection. siRNA transfection was performed to validate the effect of WIF1 on tumor invasion and the effect of treatment with genistein on tumor suppression. siRNA-WIF1 was designed and synthesized by Shanghai GenePharma Co., Ltd. (Shanghai, China). For the siRNA transfection, cells were seeded onto 12-well cell culture plates at an initial density of $6 \times 10^{4}$ cells/well. When cells were $70 \%$ confluent, they were transfected with $1 \mu \mathrm{g}$ WIF1 siRNA (siWIF1 group) or nonspecific siRNA (mock group) with FlexiTube siRNA Premix transfection reagent (Qiagen, Inc., Valencia, CA, USA) according to the manufacturer's protocols. HT29 cells without any treatment served as the control group. The following siRNA sequences were used: siWIF1, 5'-CUCAUAGGAUUUGAAGAAG-3'; and nonspecific siRNA, 5'-UCACUGCGCUCGAUGCAGUTT-3'.

RT-qPCR and western blot analyses were performed to measure the interference efficiency $48 \mathrm{~h}$ following transfection. Cell invasion/migration assays were performed as a forentioned to evaluate the effect of siWIF1 transfection among the control, mock, siWIF1 and siWIF1+genistein (siWIF1 group treated with $60 \mu \mathrm{mol} / \mathrm{l}$ genstein) groups. The above experiments were performed independently.

RT-qPCR analysis. Total RNA was extracted from cells by ZR RNA MiniPrep kit (Zymo Research Corp., Irvine, CA USA), and reverse-transcribed into cDNA using a first strand cDNA kit (Sigma-Aldrich; Merck KGaA), according to the manufacturer's protocol. The following thermocycling conditions were used for the PCR: Initial denaturation for $30 \mathrm{sec}$ at $95^{\circ} \mathrm{C}$; 40 cycles of denaturation at $95^{\circ} \mathrm{C}$ for $5 \mathrm{sec}$, annealing/extension at $60^{\circ} \mathrm{C}$ for $30 \mathrm{sec}$ and final extension at $72^{\circ} \mathrm{C}$ for $10 \mathrm{~min}$. An ABI 7300 thermocycler (Applied Biosystems; Thermo Fisher Scientific, Inc.) and the SYBR Premix Ex Taq kit (Takara Biotechnology Co., Ltd., Dalian, China) were used. The quantification was identified by $2^{-\Delta \Delta \mathrm{Cq}}(13)$. Primer sequences used for the PCR are listed in Table I.

Western blot analysis. Tissues or cells were lysed by lysis buffer (50 mM Tris-Cl, $150 \mathrm{mM} \mathrm{NaCl,} 0.02 \% \mathrm{NaN} 2$, $100 \mu \mathrm{g} / \mathrm{ml}$ phenylmethanesulfonyl fluoride, $1 \mu \mathrm{g} / \mathrm{ml}$ aprotinin, and $1 \%$ Triton X-100) and the supernatant was collected. Protein concentrations were determined using a bicinchoninic acid assay (Beyotime Institute of Biotechnology, China). A total of $20 \mu \mathrm{g}$ proteins were subjected to $10 \%$ SDS-PAGE and electroblotted onto polyvinylidene fluoride membranes (PVDF). Following blocking with 5\% skimmed dried milk in PBS for $1 \mathrm{~h}$ at room temperature, blotting membranes were probed overnight at $4{ }^{\circ} \mathrm{C}$ with the following primary antibodies: Rabbit anti-WIF1 (1:2,000; ab186845; Abcam, Cambridge,
UK), anti-E-cadherin (1:500; ab15148; Abcam), anti-MMP2 (1:1,000; ab92536; Abcam), anti-MMP9 (1:1,000; ab38898; Abcam), anti-TIMP1 (1:1,000; ab61224; Abcam), anti- $\beta$-catenin (1:5,000; ab16051; Abcam), anti-C-Myc (1:1,000; ab39688; Abcam), and anti-Cyclin D1 (1:2,000; ab226977; Abcam), GAPDH (1:2,000; ab9485; Abcam). GAPDH was used for the loading control. Subsequently, the membranes were probed with the appropriate horseradish peroxidase (HRP)-conjugated secondary antibodies: Goat anti-rabbit immunoglobulin G H\&L HRP (1:5,000; ab205718 and ab6721; Abcam) for $1 \mathrm{~h}$ at room temperature. The PVDF membranes were exposed to X-ray film and immunoreactive bands were detected by enhanced chemiluminescence detection system reagents (Amersham; GE Healthcare, Chicago, IL, USA). Lab Works Image Acquisition and Analysis software (Visionworks ${ }^{\circledR}$ LS, UVP, LLC, Phoenix, AZ, USA) was used to quantify band intensities.

Statistical analysis. Data are presented as the mean \pm standard deviation of three independent experiments. Statistical analysis was performed using SPSS software (version 13.0; SPSS, Inc., Chicago, IL, USA) and data were analyzed using one-way analysis of variance, followed by Dunnett's test. $\mathrm{P}<0.05$ was considered to indicate a statistically significant difference.

\section{Results}

Low expression of WIF1 in colon cancer tissues and cell lines. To verify the biological role of WIF1 in colon cancer, the mRNA and protein expression levels of WIF1 in colon cancer tissues and colon cancer cell lines (HT29, SW620, LOVO, HCT116) were detected by RT-qPCR and western blot analyses. The expression of WIF1 in the adjacent normal colon tissues and in the colon epithelial cell line NCM460 was detected as the control. The results demonstrated that the mRNA and protein expression of WIF1 was decreased in colon cancer tissues compared with the adjacent normal tissues (both $\mathrm{P}<0.01$; Fig. 1A-C). The mRNA and protein expression of WIF1 was additionally downregulated in colon cancer cells (HT29, SW620, LOVO and HCT116), compared with normal colon NCM460 cells (Fig. 1D-F). The above results indicated that downregulated expression of WIF1 may be associated with the initiation and/or progression of colon cancer. The HT29 cell line was selected for subsequent experiments as it exhibited the lowest expression of WIF1, compared with the other cell lines included in the present study.

Effect of genistein on HT29 cell viability, and WIF1 expression and promoter methylation. The effect of genistein on HT29 cell viability was measured by CCK8 assay. Cell viability was suppressed in the genistein-treated groups in a dose-dependent manner compared with the control group (Fig. 2A). Cell viability was respectively 66, 51, 44 and $38 \%$ of the control when treated with 10, 20, 40 and $60 \mu \mathrm{mol} / 1$ genistein for $72 \mathrm{~h}($ all $\mathrm{P}<0.01)$. HT29 cells treated with 10, 20 and $60 \mu \mathrm{mol} / 1$ genistein respectively for $72 \mathrm{~h}$ were subjected to the subsequent experimentation.

RT-qPCR and western blot analyses demonstrated that the mRNA and protein expression of WIF1 notably increased in genistein-treated cells in a dose-dependent manner (Fig. 2B-D). The above results confirmed that genistein could recover the expression and function of WIF1 to inhibit cell viability. 
Table I. Primers used for reverse transcription-quantitative polymerase chain reaction.

Primer sequence

\begin{tabular}{lll}
\cline { 2 - 3 } Gene & \multicolumn{1}{c}{ Forward $\left(5^{\prime} \rightarrow 3^{\prime}\right)$} & \multicolumn{1}{c}{ Reverse $\left(5^{\prime} \rightarrow 3^{\prime}\right)$} \\
\hline WIF1 & ATCATCTTCTTAACTGGCATTGTG & AAAAATAAAAAAAACACGCT \\
E-cadherin & CTGAAGTGACTCGTAACGAC & CATGTCAGCCAGCTTCTTGAAG \\
TIMP1 & TCGTCATCAGGGCCAAGTTC & TCCACAAGCAATGAGTGCCA \\
MMP2 & ATGCAGTGGGGGCTTAAGAA & TCTGGGGCAGTCCAAAGAAC \\
MMP9 & CATCCGGCACCTCTATGGTC & CATCGTCCACCGGACTCAAA \\
$\beta$-catenin & ATAAGAGCTCCTTGTGCGGC & GGCCATGTCCAACTCCATCA \\
c-Myc & GCCACGTCTCCACACATCAG & TGGTGCATTTCGGTTGTTG \\
CyclinD1 & GCTGGAGGTCTGCGAGGA & ACAGGAAGCGGTCCAGGTAGT \\
GAPDH & TGACTTCAACAGCGACACCCA & CACCCTGTTGCTGTAGCCAAA
\end{tabular}

MMP, matrix metalloproteinase; WIF1, Wnt inhibitory factor 1; TIMP1, tissue of metalloproteinase inhibitor 1; c-Myc, c-Myc proto-oncogene protein.

As detected by MSP, following $72 \mathrm{~h}$ of treatment with 10, 20 and $60 \mu \mathrm{mol} / 1$ genistein, levels of demethylated WIF1 increased compared with the levels of methylated WIF1. These effects occurred in a dose-dependent manner, compared withthe respective control groups (Fig. 2E). The above results indicated that the role served by genistein in colon cancer may be associated with demethylation of WIF1, to recover its tumor suppressor function.

Effect of genistein on HT29 cell invasion and migration. The invasive and migratory ability of HT29 cells treated with genistein was identified by Transwell assay. The results of the assay demonstrated that the invasive/migratory abilities of cells treated with 10, 20 and $60 \mu \mathrm{mol} / 1$ genistein for $72 \mathrm{~h}$ decreased notably in a dose-dependent manner, compared with the control group. Invasion rates in groups treated with 10, 20 and $60 \mu \mathrm{mol} / \mathrm{l}$ genistein were $\sim 75,66$ and 58\%, respectively, compared with the control group (Fig. 3A and B). Migration rates in groups treated with 10,20 and $60 \mu \mathrm{mol} / 1$ genistein were $\sim 82,69$ and $53 \%$, respectively, compared with the control group (Fig. 3C and D).

Effect of genistein on the expression of factors associated with cell invasion and the Wnt/ $\beta$-catenin signaling pathway in HT29 cells. RT-qPCR and western blot analyses were performed to detect the mRNA and protein expression of invasion/migration-associated factors, including E-cadherin, MMP2, MMP9 and TIMP1, and factors associated with the Wnt/ $\beta$-catenin signaling pathway: $\beta$-catenin, c-Myc and cyclin D1.

The results of the present study demonstrated that the mRNA and protein expression levels of E-cadherin and TIMP1 increased significantly, while the expression levels of MMP2 and MMP9 decreased significantly, in a dose-dependent manner in genistein-treated cells $(72 \mathrm{~h})$, compared with the control group (Fig. 4A-C). A significant dose-dependent decrease in mRNA and protein expression levels of $\beta$-catenin, c-Myc and cyclin D1 was observed in cells treated with genistein, compared with the control group (Fig. 4D-F). The results of the present study indicated that the inhibitory properties of genistein on HT29 cell invasion and migration may be associated with the expression of invasion/migration-associated factors, including E-cadherin, MMP2, MMP9 and TIMP1, and factors associated with the Wnt/ $\beta$-catenin signaling pathway ( $\beta$-catenin, $\mathrm{c}-\mathrm{Myc}$ and cyclin D1).

Effect of WIF1-siRNA transfection on HT29 cell invasion and migration. WIF1 knockdown and the interference efficiency were identified using RT-qPCR and western blot analyses. Transfection of siRNA-WIF1 resulted in a significant decline in WIF1 mRNA and protein expression levels in the siRNA-WIF1 group compared with the control and mock groups, which confirmed that siRNA-WIF1 was effective in silencing the expression of WIF1 (all $\mathrm{P}<0.01$; Fig. 5A-C).

The invasive and migratory abilities of the siWIF1, siWIF1+genistein, mock and HT29 control groups were identified by Transwell assay. The results of the above experiment demonstrated that the invasive/migratory abilities of the siWIF1and siWIF1+genistein groups increased significantly, compared with the control group and mock group (all $\mathrm{P}<0.05$ ). The invasion rate of the siWIF1 and siWIF1+genistein groups was 139 and $122 \%$, respectively, compared with the control group (Fig. 5D and E). The migration rates of the siWIF1 and siWIF1+genistein groups were 142 and $125 \%$, respectively, compared with the control group (Fig. 5F and G). It was confirmed that genistein was able to partly reverse the function of siWIF1 in promoting cell invasion/migration.

\section{Discussion}

Colon cancer is one of the most common malignant carcinomas, demonstrating elevated invasion and migration rates (10). DNA methylation of $\mathrm{CpG}$ islands inpromoter regions of tumor suppressor genes is considered to be an epigenetic mechanism underlying cancer development (11). Abnormal activation of the Wnt signaling pathway could induce tumor development (12). Previous studies reported that silencing of WIF1 via methylationis associated with colon cancer $(5,14,15)$. 
A

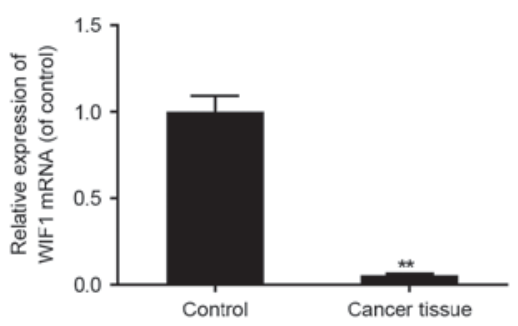

D

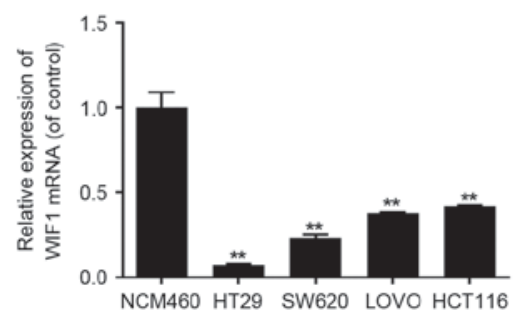

B

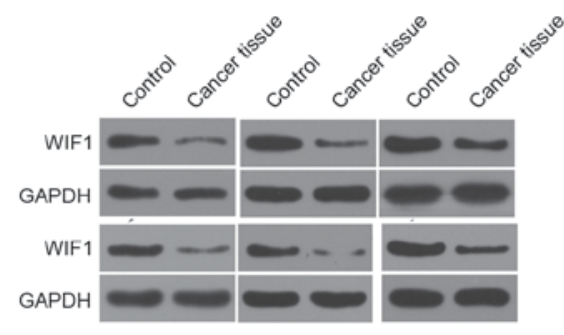

E

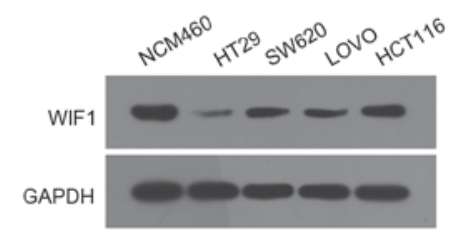

C

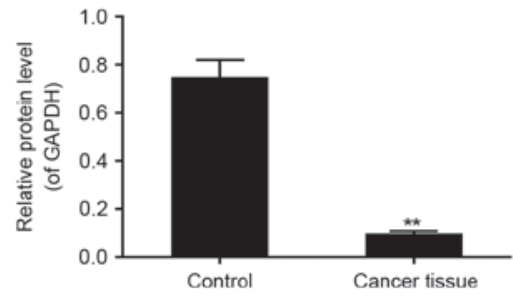

F

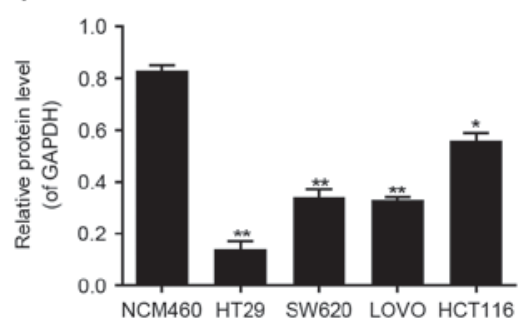

Figure 1. WIF1 expression levels in colon cancer tissues and cell lines. (A) WIF1 mRNA expression in colon cancer tissue and the adjacent normal tissue. (B) Representative western blot images of WIF1 protein expression. (C) Protein expression levels of WIF1 in colon cancer tissue and adjacent normal tissue. (D) WIF1 mRNA expression levels in the normal colon mucosa epithelial cell line NCM460 and colon cancer cell lines, including HT29, SW620, LOVO and HCT116. Protein expression levels of WIF1 were determined by (E) western blot analysis and (F) quantitative analysis in NCM460, HT29, SW620, LOVO and HCT116cell lines. GAPDH served as a loading control. Data are presented as the mean \pm standard deviation. $n=6$. ${ }^{*} \mathrm{P}<0.05$ and ${ }^{* *} \mathrm{P}<0.01$ vs. respective control group. WIF1, Wnt inhibitory factor 1.

A

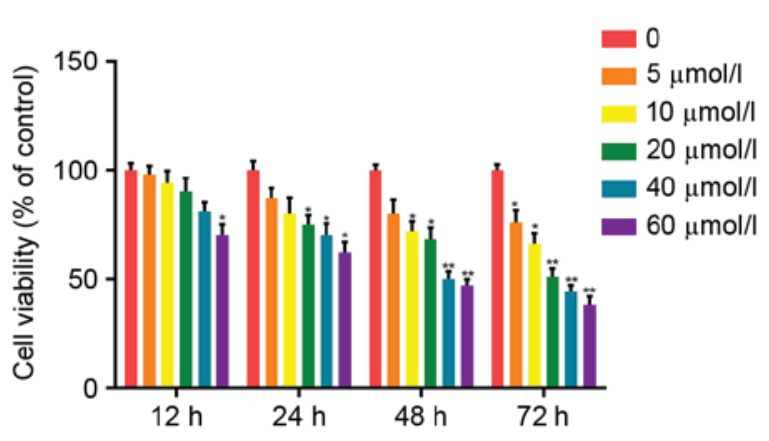

C

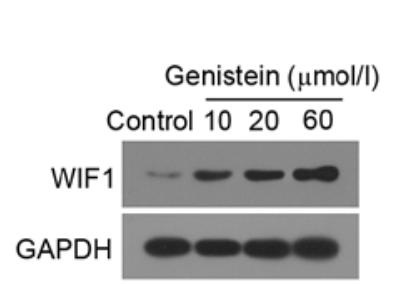

D

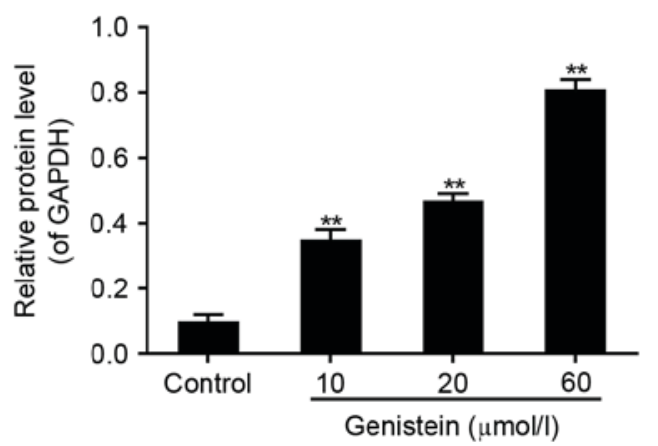

B

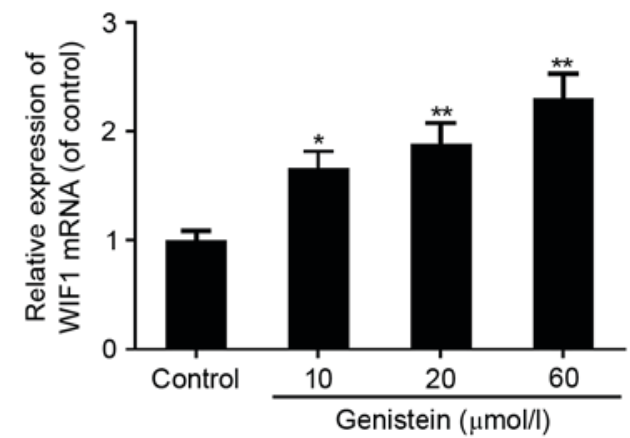

$\mathrm{E}$

Figure 2. Effect of genistein on HT29 cell viability. (A) Following treatment with 0, 5, 10, 20, 40 and $60 \mu \mathrm{mol} / 1$ genistein for 12, 24, 48 and $72 \mathrm{~h}$, a Cell Counting Kit-8 assay was performed to verify the viability of HT29 cells. (B) mRNA expression levels of WIF1 in HT29 cells. Protein expression levels of WIF1 in HT29 cells were measured by (C) western blotting and (D) quantified. (E) Methylation specific polymerase chain reaction analysis was performed to determine the amount of methylated or unmethylated WIF1. Data are presented as the mean \pm standard deviation. $\mathrm{n}=6 .{ }^{*} \mathrm{P}<0.05$ and ${ }^{* *} \mathrm{P}<0.01$ vs. respective control group. WIF1, Wnt inhibitory factor 1.

The primary aim of the present study was to elucidate the molecular mechanism underlying genistein-mediated inhibition of invasion and migration of colon cancer associated with WIF1 demethylation. Preliminary observations 
A

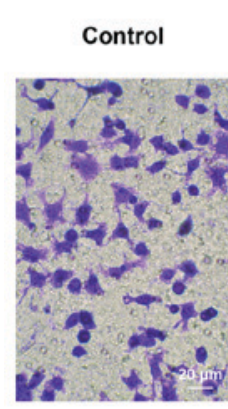

C

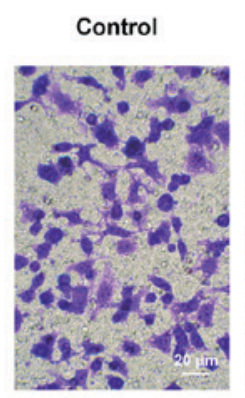

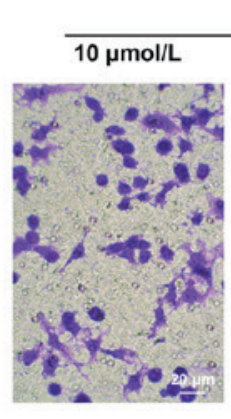
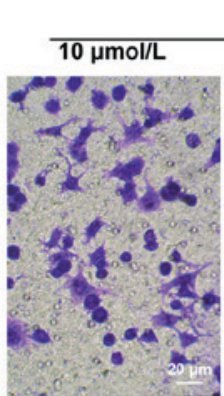

Genistein

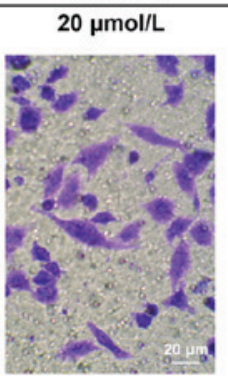

Genistein

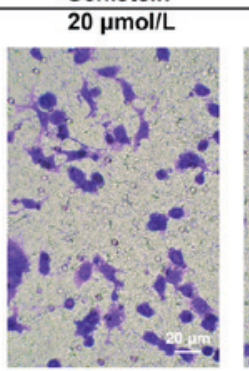

$60 \mu \mathrm{mol} / \mathrm{L}$

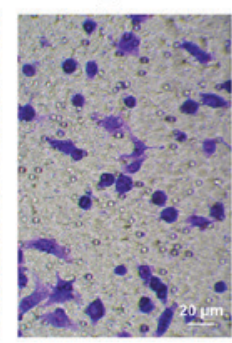

$60 \mu \mathrm{mol} / \mathrm{L}$

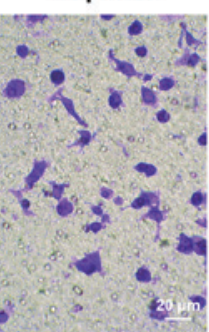

B

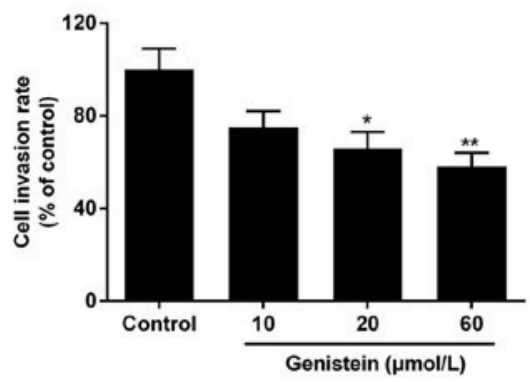

D

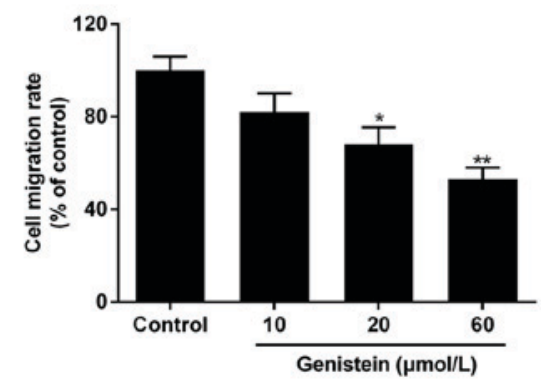

Figure 3. Effect of genistein on invasion and migration HT29 cells. Following treatment with 10, 20 and $60 \mu$ mol/1 genistein for $72 \mathrm{~h}$, (A) Cell invasion was detected and (B) quantified. Cell migration was (C) detected and (D) quantified. Data are presented as the mean \pm standard deviation. $\mathrm{n}=6$. ${ }^{*} \mathrm{P}<0.05$ and ${ }^{* *} \mathrm{P}<0.01$ vs. the control group.

A

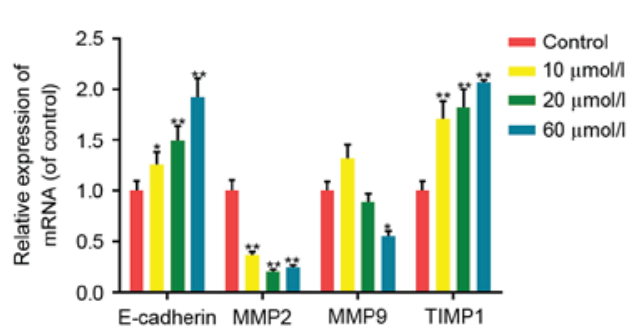

D

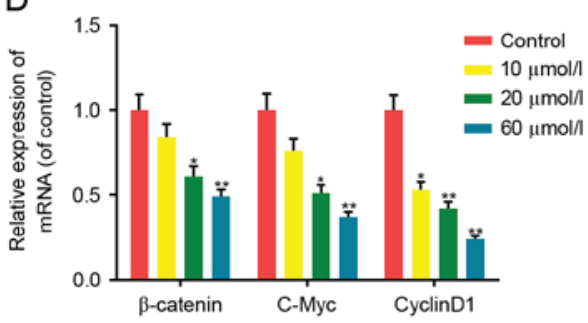

B

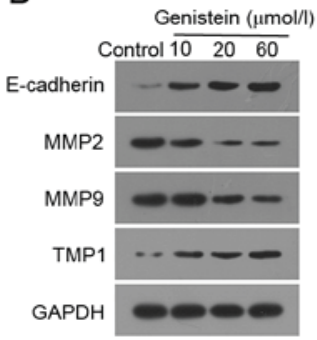

E

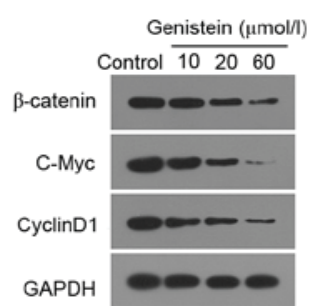

C

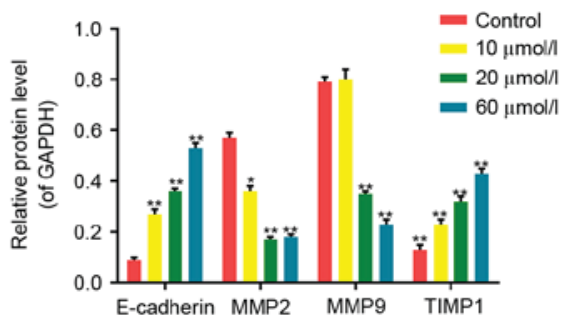

$\mathrm{F}$

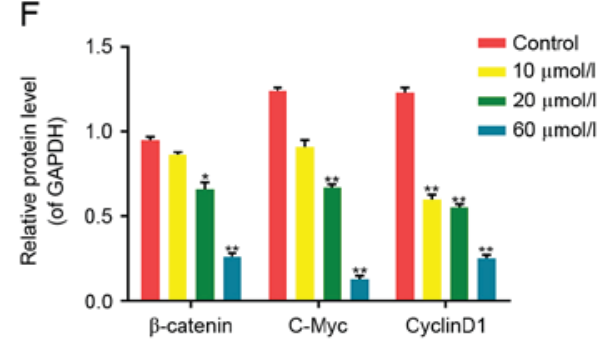

Figure 4. Effect of genistein on the expression of tumor-associated factors. Following treatment with 10, 20 and $60 \mu$ mol/1 genistein for $72 \mathrm{~h}$, the (A) mRNA and (B) protein expression of invasion/migration-associated factors (E-cadherin, MMP2, MMP9 and TIMP1) was detected in HT29 cells, and (C) quantified. The (D) mRNA and (E) protein expression of Wnt/ $\beta$-catenin-associated factors, including $\beta$-catenin, c-Myc and cyclinD1 in HT29 cells. (F) The protein levels of $\beta$-catenin, c-Myc and cyclinD1 in HT29 cells were quantified. GAPDH was detected as the control of sample loading. Data were presented as the mean \pm standard deviation. $\mathrm{n}=6 .{ }^{*} \mathrm{P}<0.05$ and ${ }^{* *} \mathrm{P}<0.01$ vs. the control group. MMP, matrix metalloproteinase; WIF1, Wnt inhibitory factor 1 ; TIMP1, tissue of metalloproteinase inhibitor 1; c-Myc, c-Myc proto-oncogene protein.

demonstrated that the expression of WIF1 decreased markedly in colon cancer tissues and cells, including HT29, SW620, LOVO and HCT116 at different rates. The expression levels of WIF1 decreased in HCT116, SW620 and LOVO cells compared with the control. The HT29 cell line demonstrated the lowest expression levels of WIF1 compared with all other cells and, therefore, was selected for further analysis. Future studies could investigate the mechanism underlying the association between genistein and cancer in order to verify the results obtained in the present study. 

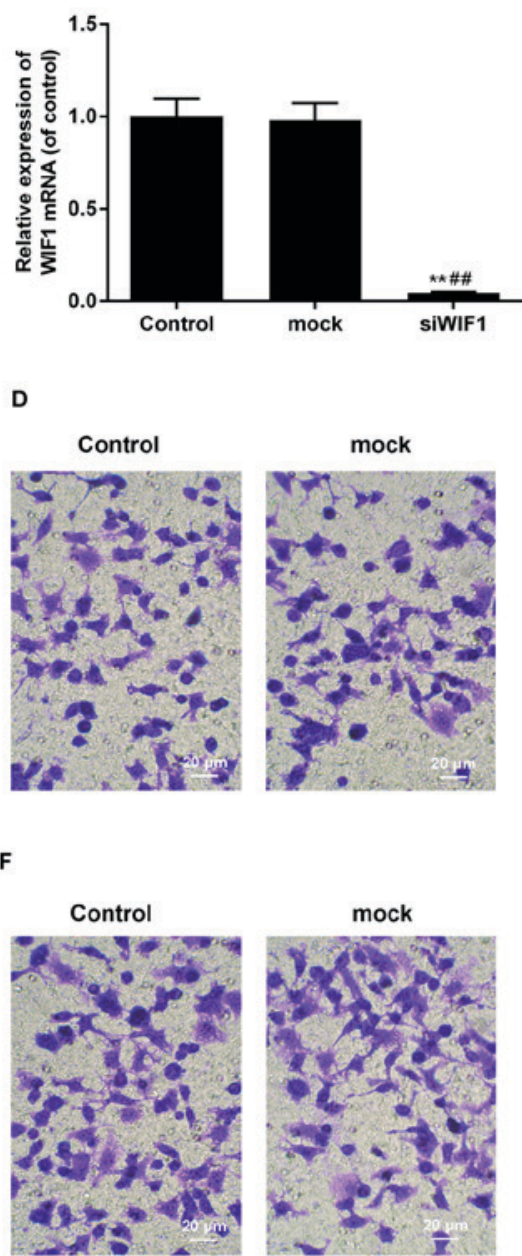

B

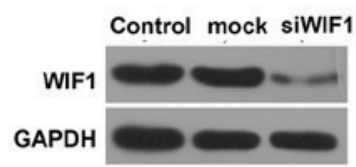

C
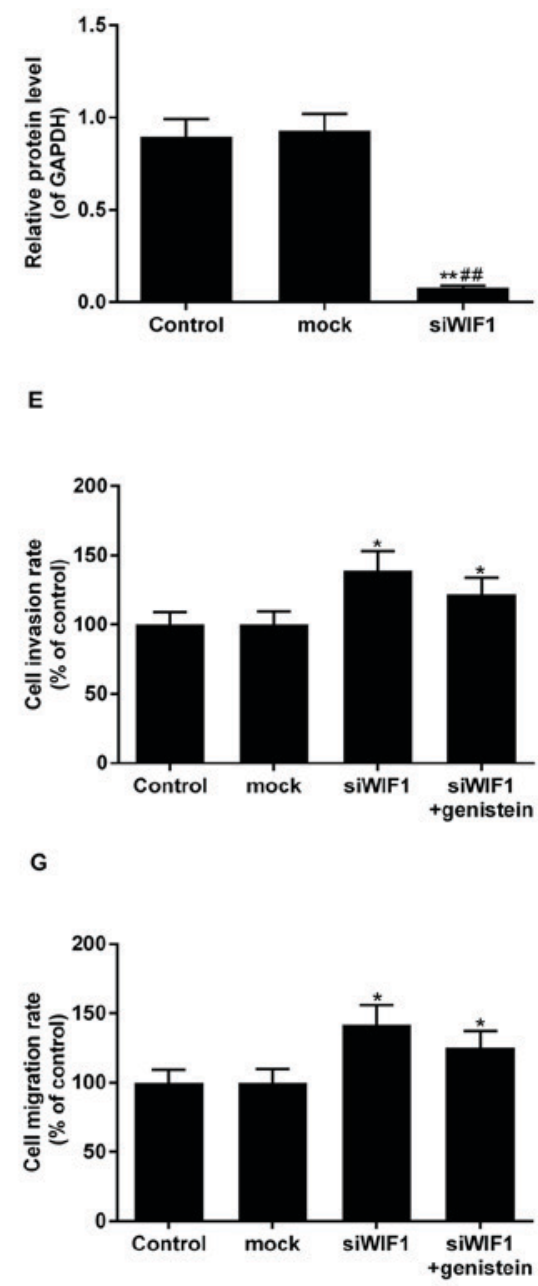

Figure 5. Effect of WIF1-siRNA transfection on WIF1 expression in HT29 cells. (A) Following transfection with siRNA-WIF1 for 48 h, the mRNA expression of WIF1 was quantified. (B) Protein levels of WIF1 in HT29 cells were measured by (B) western blot analysis and (C) quantified. The invasion ability of the siWIF1 and siWIF1+genistein groups identified by Transwell assay was (D) visualized and (E) quantified. The migration ability of the siWIF1 and siWIF1+genistein groups identified by Transwell assay was $(\mathrm{F})$ visualized and $(\mathrm{G})$ quantified. Data are presented as the mean \pm standard deviation. $\mathrm{n}=6$. ${ }^{*} \mathrm{P}<0.05$ and ${ }^{* *} \mathrm{P}<0.01$ vs. the control group, ${ }^{\# \#} \mathrm{P}<0.01$ vs. the mock group. siRNA, small interfering RNA.

Low expression of WIF1 may induce abnormal activation of the Wnt signaling pathway, promoting cell proliferation and resulting in tumor development $(16,17)$. In the present study, the proliferation of HT29 colon cancer cells treated with genistein was inhibited in a dose-dependent manner. The mRNA and protein expression levels of WIF1 increased in HT29 cells treated with genistein in a dose-dependent manner, suggesting that the function of genistein on cell proliferation was associated with the regulation of WIF1 expression. In order to further elucidate the mechanism underlying the modulation of expression of WIF1 in colon cancer cells, an MSP assay was performed to evaluate methylation levels in the promoter region of the WIF1 gene. Decreased methylation levels in the group treated with genistein, compared with the control group indicated that genistein-mediated upregulation of WIF1 expression maybe associated with the demethylation of $\mathrm{CpG}$ islands.

A number of factors are involved in the process of tumor development. The Transwell assay performed in the present study demonstrated that genistein inhibited the migration and invasion of HT29 cells in a dose-dependent manner.
It was additionally demonstrated that the mechanism underlying the above observations was associated with the regulation of invasion/migration-associated factors. MMP is a family of enzymes regulating the proteolysis of extracellular matrix-associated structural proteins, dysregulation of MMPs in cells can result in the degradation of the histological barrier that normally prevents cell invasion and migration (18-20). MMPs serve a number of roles in tumor cell invasion and migration, and have been investigated in a recent study (21). Type IV collagenases are a sub-family of MMPs that includes two members, glycosylated MMP9 and non-glycosylated MMP2. Previous studies demonstrated that Type IV collagenases are implicated in tumor cell invasion and migration processes $(22,23)$. MMPs are controlled by endogenous tissue specific inhibitors, also known as tissue inhibitors of metalloproteinases (TIMPs) (24). TIMP1 can inhibit tumor progression by suppressing MMP9-mediated release of vascular endothelial growth factor from the matrix. E-cadherin, a family member of $\mathrm{Ca}^{2+}$-dependent transmembrane glycoproteins, is an important tumor suppressor participating in intercellular signal transduction (25). 
E-cadherin is able to conjugate to $\beta$-catenin in the cytoplasm and inhibit its function in the Wnt signaling pathway. The results of the present study demonstrated that treatment with genistein resulted in decreased expression of MMP9 and MMP2, and increased expression of TIMP1 and E-cadherin in HT29 cells, compared with the control group. The above results indicated that genistein regulates tumor invasion and migration by influencing the expression levels of MMP2, MMP9, TIMP1 and E-cadherin.

The Wnt/ $\beta$-catenin signaling pathway is involved in growth-associated processes and tumor development. WIF1 is an inhibitor of the Wnt/ $\beta$-catenin signaling pathway and, therefore, silencing of WIF1 may activate the pathway and induce tumor development (26). $\beta$-catenin is a scaffold protein linking the cytoplasmic tail of classical cadherins of the endothelium to the actin cytoskeleton. Upregulation of $\beta$-catenin may cause tumor metastasis (27). Cyclin D1 is a cell cycle-related factor associated with G1/S stage transition. C-Myc encodes phosphorylated proteins, which facilitate cell proliferation and differentiation (28). Cyclin D1 and c-Myc are downstream target genes of the Wnt/ $\beta$-catenin signaling pathway (29). The mRNA and protein expression of $\beta$-catenin, $c-M y c$ and cyclin D1 decreased in HT29 cells treated with genistein, compared with the control group. It may be hypothesized that demethylation of WIF1 inhibited tumor progression by downregulating the expression of factors associated with the $\mathrm{Wnt} / \beta$-catenin signaling pathway ( $\beta$-catenin, c-Myc and cyclin D1).

Following transfection with WIF1-siRNA, the mRNA and protein levels of WIF1 in siWIF1 cells decreased markedly. The invasive and migratory abilities of the siWIF1 and siWIF1+genistein groups increased markedly compared with the control and mock groups, and genistein was able to partly reverse the function of siWIF1 in promoting cell invasion/migration.

In conclusion, the present study demonstrated that the expression of WIF1 was suppressed by methylation of $\mathrm{CpG}$ islands in the promoter region, in human colon cancer tissues and cells. Additionally, genistein recovered the expression of WIF1 in the HT29 colon cancer cell line via demethylation of WIF1, and suppressed tumor cell invasion and migration. The above observations may be associated with the regulation of cell invasion and migration-associated genes, including MMP9, MMP2, TIMP1, E-cadherin, $\beta$-catenin, c-Myc and cyclin D1. Further in vivo studies are required to confirm the results of the present study. Novel evidence may, in the future, confirm the administration of genistein to be an effective treatment for patients with colon cancer.

\section{Acknowledgements}

Not applicable.

\section{Funding}

No funding was received.

\section{Availability of data and materials}

The analysed data sets generated during the study are available from the corresponding author on reasonable request.

\section{Authors' contributions}

JZ contributed to the conception of the study. JR contributed significantly to conducting of the experiments, analysis and manuscript preparation. LT performed the data analyses and wrote the manuscript.

\section{Ethics approval and consent to participate}

Written informed consent was obtained from all participants prior to the present study. All tissue samples of patients were collected according to procedures approved by the institutional review board of the independent ethics committee of Changzhou No. 2 People's Hospital (Changzhou, China).

\section{Consent for publication}

All participants provided written informed consent prior to the present study.

\section{Competing interests}

The authors declare that they have no competing interests.

\section{References}

1. Xu S, Yao H, Pei L, Hu M, Li D, Qiu Y, Wang G, Wu L, Yao H, Zhu Z and Xu J: Design, synthesis, and biological evaluation of NAD(P)H: Quinone oxidoreductase (NQO1)-targeted oridonin prodrugs possessing indolequinone moiety for hypoxia-selective activation. Eur J Med Chem 132: 310-321, 2017.

2. Choi Y, Sateia HF, Peairs KS and Stewart RW: Screening for colorectal cancer. Semin Oncol 44: 34-44, 2017.

3. Hu J, Li J, Yue X, Wang JC, Wang JF, Liu JZ and Kong DL: Targeting BCRP/ABCG2 by RNA interference enhances the chemotherapy sensitivity of human colon cancer side population cells. J Huazhong Univ Sci Technolog Med Sci 37: 231-236, 2017.

4. Ahmed I, Roy BC, Subramaniam D, Ganie SA, Kwatra D Dixon D, Anant S, Zargar MA and Umar S: An ornamental plant targets epigenetic signaling to block cancer stem cell-driven colon carcinogenesis. Carcinogenesis 37: 385-396, 2016.

5. Patai ÁV, Valcz G, Hollósi P, Kalmár A, Péterfia B, Patai Á, Wichmann B, Spisák S, Barták BK, Leiszter $\mathrm{K}$, et al: Comprehensive DNA methylation analysis reveals a common ten-gene methylation signature in colorectal adenomas and carcinomas. PLoS One 10: e0133836, 2015.

6. Sánchez-Vega F, Gotea V, Chen YC and Elnitski L: CpG island methylator phenotype in adenocarcinomas from the digestive tract: Methods, conclusions and controversies. World J Gastrointest Oncol 9: 105-120, 2017.

7. Chan SL, Cui Y, van Hasselt A, Li H, Srivastava G, Jin H, Ng KM, Wang Y, Lee KY, Tsao GS, et al: The tumor suppressor Wnt inhibitory factor 1 is frequently methylated in nasopharyngeal and esophageal carcinomas. Lab Invest 87: 644-650, 2007.

8. Bilir B, Sharma NV, Lee J, Hammarstrom B, Svindland A, Kucuk O and Moreno CS: Effects of genistein supplementation on genome-wide DNA methylation and gene expression in patients with localized prostate cancer. Int J Oncol 51: 223-234, 2017.

9. Sundaram MK, Ansari MZ, Mutery AA, Ashraf M, Nasab R, Rai S, Rais N and Hussain A: Genistein induces alterations of epigenetic modulatory signatures in human cervical cancer cells. Anticancer Agents Med Chem: Sep 18, 2017 (Epub ahead of print).

10. Chen G, Zhou T, Li Y, Yu Z and Sun L: p53 target miR-29c-3p suppresses colon cancer cell invasion and migration through inhibition of PHLDB2. Biochem Biophys Res Commun 487: 90-95, 2017.

11. Zhang L, Wu H, Xiao X, Li K, Zhang Y, Zhang L and Wen T: Analysis on regulatory network linked to Hpa gene in invasion and metastasis of colon cancer. Saudi J Biol Sci 24: 504-507, 2017. 
12. Luo X, Xiong X, Shao Q, Xiang T, Li L, Yin X, Li X, Tao Q and Ren G: The tumor suppressor interferon regulatory factor 8 inhibits $\beta$-catenin signaling in breast cancers, but is frequently silenced by promoter methylation. Oncotarget 8: 48875-48888, 2017.

13. Livak KJ and Schmittgen TD: Analysis of relative gene expression data using real-time quantitative PCR and the 2(-Delta Delta C(T)) method. Methods 25: 402-408, 2001.

14. Wang LS, Kuo CT, Huang TH, Yearsley M, Oshima K, Stoner GD, Yu J, Lechner JF and Huang YW: Black raspberries protectively regulate methylation of Wnt pathway genes in precancerous colon tissue. Cancer Prev Res (Phila) 6: 1317-1327, 2013.

15. Afgar A, Fard-Esfahani P, Mehrtash A, Azadmanesh K Khodarahmi F, Ghadir M and Teimoori-Toolabi L: MiR-339 and especially miR-766 reactivate the expression of tumor suppressor genes in colorectal cancer cell lines through DNA methyltransferase 3B gene inhibition. Cancer Biol Ther 17: 1126-1138, 2016

16. Yang SH, Li SL, Dong ZM and Kan QC: Epigenetic inactivation of Wnt inhibitory factor-1 in human esophageal squamous cell carcinoma. Oncol Res 20: 123-130, 2012.

17. Stewart DJ: Wnt signaling pathway in non-small cell lung cancer. J Natl Cancer Inst 106: djt356, 2014.

18. Stanciu AE, Zamfir-Chiru-Anton A, Stanciu MM, Popescu CR and Gheorghe DC: Imbalance between matrix metalloproteinases and tissue inhibitors of metalloproteinases promotes invasion and metastasis of head and neck squamous cell carcinoma. Clin Lab 63: 1613-1620, 2017.

19. Das A, Monteiro M, Barai A, Kumar S and Sen S: MMP proteolytic activity regulates cancer invasiveness by modulating integrins. Sci Rep 7: 14219, 2017.

20. Shi Y, An D, Liu Y, Feng Q, Fang X, Pan G and Wang Q: Propoxur enhances MMP-2 expression and the corresponding invasion of human breast cancer cells via the ERK/Nrf2 signaling pathway. Oncotarget 8: 87107-87123, 2017.

21. Cheng Z, Limbu MH, Wang Z, Liu J, Liu L, Zhang X, Chen P and Liu B: MMP-2 and 9 in chronic kidney disease. Int J Mol Sci 18: 276, 2017.
22. Shen EY, Wang WG, Li Y, Zhang SH and Zhen YS: Inhibitory effect of endoplasmic reticulum-retained anti-type IV collagenase intrabody on invasion and proliferation of cancer cell. Ai Zheng 23: 1005-1010, 2004 (In Chinese).

23. Lu L, Li X, Xu P, Zheng Y and Wang X: Tenuigenin down-regulates the release of nitric oxide, matrix metalloproteinase- 9 and cytokines from lipopolysaccharide-stimulated microglia. Neurosci Lett 650: 82-88, 2017

24. Thiele ND, Wirth JW, Steins D, Koop AC, Ittrich H, Lohse AW and Kluwe J: TIMP-1 is upregulated, but not essential in hepatic fibrogenesis and carcinogenesis in mice. Sci Rep 7: 714, 2017.

25. Gulati N, Rathore AS, Juneja S and Rastogi P: Expression of E-cadherin and B-cell lymphoma 2 in oral cancer: A ratio-based planning for targeted therapy. Indian J Dent Res 28: 3-9, 2017.

26. Huang M, Chen C, Geng J, Han D, Wang T, Xie T, Wang L, Wang $\mathrm{Y}$, Wang $\mathrm{CH}$, Lei $\mathrm{Z}$ and Chu $\mathrm{X}$ : Targeting KDM1A attenuates $\mathrm{Wnt} / \beta$-catenin signaling pathway to eliminate sorafenib-resistant stem-like cells in hepatocellular carcinoma. Cancer Lett 398: 12-21, 2017.

27. Vega OA, Lucero CMJ, Araya HF, Jerez S, Tapia JC, Antonelli M, Salazar-Onfray F, Las Heras F, Thaler R, Riester SM, et al: Wnt $/ \beta$-catenin signaling activates expression of the bone-related transcription factor RUNX2 in select human osteosarcoma cell types. J Cell Biochem 118: 3662-3674, 2017.

28. Strindlund K, Troiano G, Sgaramella N, Coates PJ, Gu X, Boldrup L, Califano L, Fahraeus R, Muzio LL, Ardito F, et al: Patients with high c-MYC expressing squamous cell carcinomas of the tongue show better survival than those with low-and medium-expressing tumours. J Oral Pathol Med 46: 967-971, 2017.

29. Hong F, Ze Y, Zhou Y, Hong J, Yu X, Sheng L and Wang L: Nanoparticulate $\mathrm{TiO} 2$-mediated inhibition of the Wnt signaling pathway causes dendritic development disorder in cultured rat hippocampal neurons. J Biomed Mater Res A 105: 2139-2149, 2017. 